\title{
Low Pyridoxine Concentrations Enhance Lipopolysaccharide- Stimulated Gene Expression of Cyclooxygenase-2 and Inducible Nitric Oxide Synthase in RAW264.7 Cells
}

\author{
Hiroaki KANOUCHI \\ Department of Veterinary Pathobiology, Joint Faculty of Veterinary Medicine, Kagoshima University, \\ 1-21-24 Korimoto, Kagoshima 890-0065, Japan
}

(Received June 4, 2013)

\begin{abstract}
Summary Pyridoxal (PL) has been shown to suppress lipopolysaccharide (LPS)-induced gene expression of cyclooxygenase-2 (COX-2) and inducible nitric oxide synthase (iNOS). Commonly used cell culture media contain extremely high concentrations of pyridoxine (PN) compared to total serum levels of vitamin $\mathrm{B}_{6}$. Therefore, we evaluated how physiological concentrations of PN influence LPS-stimulated gene expression of COX-2 and iNOS. The mouse macrophage cell line, RAW264.7, was cultured in PN-free DMEM supplemented with $10 \%$ fetal bovine serum (DMEM(-PN+FBS)) for $7 \mathrm{~d}$. Although the level of pyridoxal 5 '-phosphate in these cells was decreased by $65 \%$, no change was observed in cell proliferation rate or aspartate aminotransferase activity for $7 \mathrm{~d}$. LPS-induced expression of COX-2 mRNA was compared between DMEM(+FBS) and DMEM(-PN+FBS). COX-2 expression was enhanced by 2.2 or 1.9 times with a 1 or $3 \mathrm{~d}$ treatment, respectively; however, no difference was observed at $7 \mathrm{~d}$. PN $(0.032-100 \mu \mathrm{M})$ added to the DMEM $(-\mathrm{PN}+\mathrm{FBS})$ and RAW264.7 cells was cultured in the medium containing each concentration of PN for $1 \mathrm{~d}$. Enhancement of COX-2 and iNOS gene expression was suppressed by PN addition in a concentration-dependent manner. COX-2 and iNOS mRNA were similarly expressed in cells grown in media containing PN at $4 \mu \mathrm{M}$ or higher. Overall, induction of COX-2 and iNOS by LPS was transiently enhanced when RAW264.7 cells were cultured in physiological PN concentrations.
\end{abstract}

Key Words vitamin $\mathrm{B}_{6}$, pyridoxine, COX-2, iNOS, NF- $\kappa \mathrm{B}$

Cyclooxygenase-2 (COX-2) and inducible nitric oxide synthase (iNOS) are related to the production of two major inflammatory mediators, prostaglandins and nitric oxide, respectively (1). These inflammatory mediators are essential for the immune system, and their secretion level is well controlled. Lipopolysaccharide (LPS) is the major component of the outer membrane of Gram-negative bacteria. LPS induces nuclear kappa $\mathrm{B}(\mathrm{NF}-\kappa \mathrm{B})$-dependent expression of iNOS and COX-2 in the mouse macrophage cell line, $\operatorname{RAW} 264.7(2,3)$.

The water soluble vitamin $\mathrm{B}_{6}\left(\mathrm{~B}_{6}\right)$ is required for normal growth, development, and metabolism in animals. Molecularly, the most important role of $\mathrm{B}_{6}$ is as a coenzyme. $\mathrm{B}_{6}$ has six forms: pyridoxine (PN), pyridoxal (PL), pyridoxamine, and their $5^{\prime}$-phosphate forms. All forms of $\mathrm{B}_{6}$ are converted to the coenzyme, pyridoxal 5'-phosphate (PLP) in cells (4). PLP is needed in the metabolism of various amino acids. Aspartate aminotransferase (AST) is one of the enzymes that depend on PLP (5). Furthermore, PLP can bind to various proteins, such as DNA polymerase (6), RNA polymerase (7), and steroid hormone receptors $(8)$, to change their properties via a Schiff base to the $\varepsilon$-amino group of a lysine residue

E-mail: kano@agri.kagoshima-u.ac.jp at the DNA binding site of the proteins. However, the physiological role of these binding actions of PLP is not well understood. Yanaka et al. (2) reported that PL suppresses LPS-mediated activation of NF- $\kappa \mathrm{B}$ via the inhibition of inhibitor of kappa B ( $\mathrm{\kappa} \kappa \mathrm{B})$ degradation, and that PLP converted from PL may bind the Lys residue of the polyubiquitin site of $\mathrm{I} \kappa \mathrm{B}$.

Dulbecco's Modified Eagle Medium (DMEM) contains a high concentration of $\mathrm{PN}(20 \mu \mathrm{M}$, or $3,382 \mu \mathrm{g} / \mathrm{L})$ as a source of PLP compared with physiological concentrations of total $\mathrm{B}_{6}$ in serum, found to be at $40.5 \pm 12.0 \mu \mathrm{g} / \mathrm{L}$ in individuals not taking drugs or vitamins (9). This discrepancy thus raises concerns for previous studies using DMEM for cell culture, because these studies may have been performed under different conditions than those in vivo. Therefore, in the present study we examined how a low concentration of PN influences LPS-induced expression of iNOS and COX-2 in RAW264.7 cells.

\section{Materials and Methods}

Cell culture. RAW264.7 (American Type Culture Collection, Manassas, VA) cells were cultured in DMEM supplemented with $10 \%$ fetal bovine serum (FBS) and maintained in a $5 \% \mathrm{CO}_{2}$ humidified incubator at $37^{\circ} \mathrm{C}$. Before reaching confluence, cells were detached us- 
ing $0.05 \%$ trypsin-0.5 mM ethylenediaminetetraacetic acid (trypsin-EDTA) and re-plated in new $10 \mathrm{~cm}$ tissue culture dishes (Iwaki, Tokyo, Japan). The medium was changed every $3 \mathrm{~d}$. To evaluate viable and dead cell numbers, cells were incubated with $10 \%(\mathrm{v} / \mathrm{v})$ trypan blue and counted using a hemocytometer. Cell proliferation was evaluated by the trypan blue assay and Alamar blue assay (10). Cells were seeded at $1 \times 10^{5}$ cells on $6 \mathrm{~cm}$ dishes (Iwaki) or $6 \times 10^{3}$ cells/well in 96-well plates (Iwaki) for the trypan blue assay or Alamar blue assay, respectively. To determine AST activity and PLP concentration, cells were seeded at $1 \times 10^{6}$ in $10 \mathrm{~cm}$ tissue culture dishes (Iwaki).

Treatments. PN-free DMEM was purchased from Funakoshi (Tokyo, Japan). PN-HCl (Wako Pure Chemical Industries, Ltd., Osaka, Japan) was dissolved to $0.5 \mathrm{M}$ in $\operatorname{DMEM}(-\mathrm{PN}+\mathrm{FBS})(\mathrm{pH} 7.4)$, and diluted to 0, 0.032, $0.16,0.8,4,20$, and $100 \mu \mathrm{M}$ in $\operatorname{DMEM}(-\mathrm{PN}+\mathrm{FBS})$. To induce COX-2 and iNOS, RAW264.7 cells were stimulated with LPS ( $1 \mu \mathrm{g} / \mathrm{mL}$, Wako) for $4 \mathrm{~h}$ before collecting total RNA from the cells.

Cell proliferation. Dispersed cells from $6 \mathrm{~cm}$ dishes were counted every day for $7 \mathrm{~d}$. Cell proliferation rate was also evaluated with the Alamar blue assay. Briefly, cells in a 96-well plate were incubated (3 h) with $10 \%$ (v/v) AlamarBlue (Trek Diagnostic Systems, Cleveland, $\mathrm{OH}$ ), every day for $7 \mathrm{~d}$, and absorbance (at $570 \mathrm{~nm}$ ) was measured using a plate reader (Sunrise Thermo, Tecan, Lausanne, Switzerland).

Measurement of AST activity and PLP concentration. RAW264.7 cells were cultured in DMEM(-PN+FBS) for 1 , 3, or $7 \mathrm{~d}$. After cells were rinsed (using phosphate buffered saline [PBS]) they were scraped with a rubber scraper. Collected cells were centrifuged $(300 \times g$, $3 \mathrm{~min}$ ), and immediately homogenized $(5 \mathrm{~s}$ in $0.5 \mathrm{~mL}$ of cold PBS) using a PT-2100 Polytron (Kinematica AG, Lucerne, Switzerland) at maximum speed. Protein concentration was determined from cell lysates using the BCA protein assay kit (Pierce, Rockford, IL). AST activity from cell lysates was determined by the Fuji DRI-CHEM Slide GOT/AST-PIII (Fujifilm, Tokyo, Japan), according to the manufacturer's instructions. This product was used because it doesn't contain any $\mathrm{B}_{6}$, which would otherwise affect the results given by the AST assay. Analyses of PLP concentrations were performed according to methods described previously (3). PLP concentrations were normalized against total protein concentration.

Quantitative real time polymerase chain reaction. RAW264.7 cells were cultured in $\operatorname{DMEM}(-\mathrm{PN}+\mathrm{FBS})$ for 1,3 or $7 \mathrm{~d}$, and after each time-exposure, cells were stimulated with LPS for $4 \mathrm{~h}$, then total RNA was extracted. Total RNA was prepared from the addition of TRIzol reagent (Invitrogen, Tokyo, Japan) on the cells, according to the manufacturer's protocol. cDNA was synthesized with a PrimeScript RT Reagent Kit (Takara, Tokyo, Japan). Quantitative realtime PCR analyses were performed using a Lightcycler 1.5 (Roche Diagnostics, Tokyo, Japan) and SYBR Premix Ex Taq (Takara). The primer sets for cyclooxygenase-2 (COX-2), inducible nitric oxide synthase

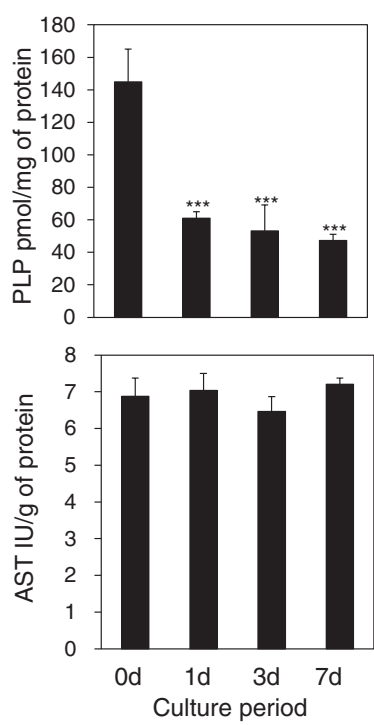

Fig. 1. Pyridoxal 5'-phosphate (PLP) concentration and AST activity. RAW264.7 cells in pyridoxine (PN)free DMEM supplemented with $10 \%$ FBS for $0,1,3$ or $7 \mathrm{~d}$. PLP concentration (upper panel) and AST activity (lower panel) were normalized against total protein concentration. ${ }^{* * *} p<0.001$ vs. $0 \mathrm{~d}$.

(iNOS), and glyceraldehyde-3-phosphate-dehydrogenase (GAPD) were purchased from Takara (COX-2, 5'-GCCAGGCTGAACTTCGAAACA-3' and 5'-GCTCACGAGGCCACTGATACCTA-3'; iNOS, 5'-AAACTGTGTCCTGGAGGTTCTG-3' and 5'-CAAGGCCTCCAATCTCTGCCTA-3'; GAPD, 5'-AAATGGTGAAGGTCGGTGTG-3' and 5'-TGAAGGGGTCGTTGATGG-3'). The cycling parameters were an initial step at $95^{\circ} \mathrm{C}$ for $10 \mathrm{~s}$, followed by 40 cycles of $95^{\circ} \mathrm{C}$ for $5 \mathrm{~s}, 60^{\circ} \mathrm{C}$ for $10 \mathrm{~s}$ and $72^{\circ} \mathrm{C}$ for $10 \mathrm{~s}$. Expression levels of COX-2 mRNA levels were normalized by GAPD mRNA levels. Data are representative of three separate mRNA isolations.

Statistical analysis. All data are expressed as the mean \pm SD from three independent experiments, and were analyzed by one-way ANOVA followed by the Dunnett post hoc test using Statistical Package for the Social Sciences software ver. 21 (International Business Machines Corporation, Armonk, NY). Significance was reached at values of $p<0.05$ or $p<0.001$.

\section{Results and Discussion}

Cell growth and AST activity of RAW264.7 cells under low PN conditions

Total $\mathrm{B}_{6}$ levels $(43.5 \pm 13.4 \mathrm{ng} / \mathrm{mL})$ have been found in fetal calf serum, which is commonly used for cell culturing (9). If all $\mathrm{B}_{6}$ was estimated to be $\mathrm{PN}$, the concentration of $\mathrm{B}_{6}$ would therefore correspond to $257 \mathrm{nM}$ of PN. Therefore, the estimated concentration of $\mathrm{PN}$ in $\operatorname{DMEM}(-\mathrm{PN})$ supplemented with $10 \% \mathrm{FBS}$ is approximately $26 \mathrm{nM}$. Under normal culture conditions using PN containing DMEM, the PLP level was detected as $145 \pm 12 \mathrm{pmol} / \mathrm{mg}$ of protein (Fig. 1 upper panel). This level was significantly reduced to $61 \pm 7$, $53 \pm 9$, and $47 \pm 2 \mathrm{pmol} / \mathrm{mg}$ of protein at 1,3 , and 


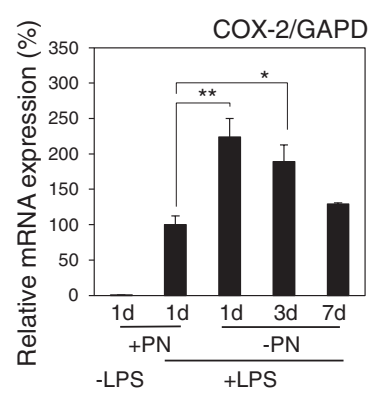

Fig. 2. Transient enhancement of LPS-stimulated COX-2 mRNA expression under low PN concentration. RAW264.7 cells were cultured in PN-free DMEM supplemented with $10 \%$ FBS for 1,3 or $7 \mathrm{~d}$ before stimulation with LPS $(1 \mu \mathrm{g} / \mathrm{mL}, 4 \mathrm{~h})$. Gene expression of COX-2 or GAPD was determined with realtime PCR. ${ }^{*} p<0.05,{ }^{* *} p<0.01$. PN, pyridoxine; LPS, lipopolysaccharide; COX-2, cyclooxygenase-2; GAPD, glyceraldehyde-3-phosphate-dehydrogenase.

$7 \mathrm{~d}$, respectively after normal media was replaced with $\operatorname{DMEM}(-\mathrm{PN}+\mathrm{FBS})$. We evaluated whether the reduction of PLP affects AST activity and the cell proliferation. AST activity didn't change under DMEM(-PN+FBS) conditions (Fig. 1 lower panel). Both assays (trypan blue exclusion and Alamar blue assay) revealed normal proliferation of cells under DMEM(-PN+FBS) (data not shown). Taken together, these results showed that RAW264.7 cells survive in DMEM(-PN+FBS). Furthermore, $10 \%$ FBS in PN-free DMEM may be enough to supply $\mathrm{B}_{6}$ to maintain RAW264.7 cells.

Effect of PN-free medium on the expression of COX-2 mRNA

We next examined whether LPS-mediated induction of COX-2 is affected under DMEM(-PN+FBS) (Fig. $2)$. LPS enhanced the expression of COX-2 mRNA by 100 -fold ( $p<0.001$ vs. without LPS condition). LPSmediated induction of COX-2 mRNA increased by 2.2fold in cells cultured for $1 \mathrm{~d}$ in DMEM(-PN+FBS) but decreased to 1.9- and 1.3-fold at 3 and $7 \mathrm{~d}$, respectively. The mechanism underlying the transient increase is unclear. Although cells may adapt to low $\mathrm{B}_{6}$ concentrations, further experiments are required to elucidate the relationship between low $\mathrm{B}_{6}$ concentrations and COX-2 induction. We next sought to examine the minimum effective concentration of $\mathrm{PN}$ on the enhancement of gene expression of COX-2 and iNOS. Cells were cultured for $1 \mathrm{~d}$ in each PN concentration, which was prepared in DMEM(-PN+FBS). The levels of expression of these 2 genes of each concentration of PN were compared to those of $20 \mu \mathrm{M}$ of PN because normal DMEM contains $20 \mu \mathrm{M}$ PN (Fig. 3). Cells cultured without PN exhibited the highest increase of LPS-mediated induction of COX-2 and iNOS mRNA, which was suppressed with PN in a concentration-dependent manner. mRNA expression of COX-2 and iNOS was similarly reduced by $4 \mu \mathrm{M}$ PN compared with that of $20 \mu \mathrm{M}$ PN (Fig. 3). Because the physiological concentration of $\mathrm{B}_{6}$ is similar that of PN $(0.16 \mu \mathrm{M})(9)$, the expression level of COX-2 and iNOS may thus be influenced by changing the plasma
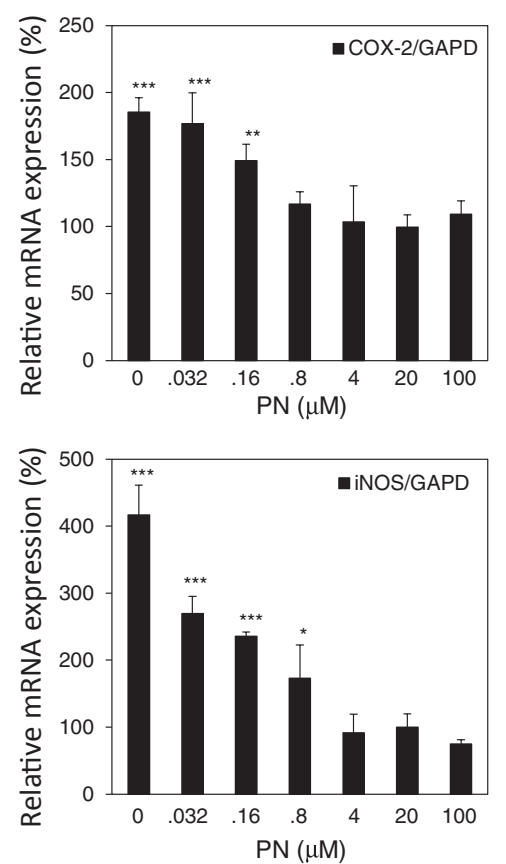

Fig. 3. PN suppresses LPS-stimulated expression of COX-2 and iNOS mRNA. RAW264.7 cells were cultured with PN in PN-free DMEM for $1 \mathrm{~d}$ before stimulation with LPS $(1 \mu \mathrm{g} / \mathrm{mL})$ for $4 \mathrm{~h}$. Gene expression for COX2 , iNOS, or GAPD was determined by real-time PCR. ${ }^{*} p<0.05,{ }^{* *} p<0.01,{ }^{* * *} p<0.001$ vs. $20 \mu \mathrm{M}$ of PN. PN, pyridoxine; LPS, lipopolysaccharide; COX-2, cyclooxygenase-2; iNOS, inducible nitric oxide synthase; GAPD, glyceraldehyde-3-phosphate-dehydrogenase.

concentration of $\mathrm{B}_{6}$.

\section{Conclusion}

Intracellular levels of PLP decreased when RAW264.7 cells were cultured in DMEM $(-\mathrm{PN}+\mathrm{FBS})$ but cell function was unaffected because the cell proliferation rate and AST activity did not change. However, LPS increased the expression of COX-2 mRNA by 2.2 times when cells were cultured in DMEM( $(-\mathrm{PN}+\mathrm{FBS})$ for $1 \mathrm{~d}$. Enhanced expression of COX-2 and iNOS genes was suppressed by PN in a concentration-dependent manner. The expression of COX-2 and iNOS is higher under the physiological concentration of PN rather than that commonly used in DMEM.

\section{REFERENCES}

1) Kim SF, Huri DA, Snyder SH. 2005. Inducible nitric oxide synthase binds, S-nitrosylates, and activates cyclooxygenase-2. Science 310: 1966-1970.

2) Yanaka N, Koyama TA, Komatsu S, Nakamura E, Kanda M, Kato N. 2005. Vitamin $B_{6}$ suppresses NF-kappaB activation in LPS-stimulated mouse macrophages. Int $J$ Mol Med 16: 1071-1075.

3) Kanouchi H, Shibuya M, Tsukamoto S, Fujimura Y, Tachibana H, Yamada K, Oka T. 2010. Comparisons of uptake and cell surface binding among pyridoxal, pyridoxine, and pyridoxamine in RAW264.7 cells. Nutrition 26: $648-652$.

4) Sakurai T, Asakura T, Matsuda M. 1988. Transport and 
metabolism of pyridoxine in the intestine of the mouse. $J$ Nutr Sci Vitaminol 34: 179-187.

5) Shibuya M, Okada M. 1986. Effect of pyridoxine-deficiency on the turnover of aspartate aminotransferase isozymes in rat liver. J Biochem 99: 939-944.

6) Diffley JF. 1988. Affinity labeling the DNA polymerase alpha complex. I. Pyridoxal 5'-phosphate inhibition of DNA polymerase and DNA primase activities of the DNA polymerase alpha complex from Drosophila melanogaster embryos. J Biol Chem 263: 14669-14677.

7) Martial J, Zaldivar J, Bull P, Venegas A, Valenzuela P. 1975. Inactivation of rat liver RNA polymerases I and II and yeast RNA polymerase I by pyridoxal 5'-phosphate.
Evidence for the participation of lysyl residues at the active site. Biochemistry 14: 4907-4911.

8) Allgood VE, Cidlowski JA. 1992. Vitamin $\mathrm{B}_{6}$ modulates transcriptional activation by multiple members of the steroid hormone receptor superfamily. J Biol Chem 267: 3819-3824.

9) Baker H, DeAngelis B, Frank O. 1988. Vitamins and other metabolites in various sera commonly used for cell culturing. Experientia 44: 1007-1010.

10) Goegan P, Johnson G, Vincent R. 1995. Effects of serum protein and colloid on the AlamarBlue assay in cell cultures. Toxicol In Vitro 9: 257-266. 